\title{
Research on reactive power optimization of district power network with large-scale new energy
}

\author{
Wang Dan ${ }^{\mathrm{a}}$, Tong Jingjing ${ }^{\mathrm{b}}$, Zhang Jianhua ${ }^{\mathrm{c}}$ \\ State Key Laboratory of Alternate Electrical Power System with Renewable Energy Sources, North \\ China Electric Power University, Beijing 102206, China \\ aemail:sarielwd@163.com, bemail:christine_tong2013@163.com, cemail:Jhzhang001@163.com
}

Keywords: Large scale new energy; Dynamic reactive power optimization; Three-level optimization

\begin{abstract}
Considering the voltage problems of district power network with large-scale new energy, the reactive power optimization model is discussed in this paper, and the renewable power plants are taken as continuous reactive power adjustment means to participate in the optimization. From two time scales of day-ahead and real-time, a three-level dynamic optimization model for coordinated control of district power network and new energy station is built and different objective functions are constructed of each level according to different needs and control variables. Finally, a case of district power network is used to validate the proposed model, and the results show that the model can ensure the safe operation of the grid at different load levels.
\end{abstract}

\section{Introduction}

For reactive power optimization problems in district power network with new energy generation, reference [1-2] considered the DFIG wind farm's reactive power regulation ability and studied the control strategy of reactive power-voltage from the perspective of global optimization. For dynamic reactive power optimization modeling, reference [3] studied the day-ahead scheduling optimization model for capacitors and transformer taps but the solving process was complex. The literatures above only considered one time section or day-ahead optimization, lacking of the description of scheduling operation's process throughout the whole day, coordination optimization of capacitors and new energy output and the interaction between power grid and new energy station.

This paper assumed the situation where the installed capacity of new energy generation is greater than the local load demand. To solve the voltage problem of $110 \mathrm{kV}$ district network, a three-level reactive power optimization model was proposed from a hierarchical perspective. Every power plant was taken as an integral whole to participate in the grid reactive power optimization, and the plant implemented optimization inside itself. In order to coordinate the new energy station's output and capacitors, the grid control was divided into a bi-level optimization of day-ahead and real-time control considering the limit of action times of capacitors.

\section{Framework of three-level reactive power optimization}

The framework of proposed optimization model is shown in Fig.1. From the perspective of day-ahead and real-time, the process of reactive power optimization in regional grid was described.

(1)First-level optimization considering discrete reactive power conditioning equipment: according to the load forecast, new energy station output forecast and reactive power output range, optimize the next day's capacitor action sequence and switching capacity.

(2)Second-level optimization considering continuous reactive power source: real-time optimization with control variables of new energy stations' reactive power output. The response speed of continuous reactive power source in new energy stations is fast enough to meet the demand of real-time scheduling.

(3) Third-level optimization in new energy station: according to the scheduling instruction, the control system of wind power unit or photovoltaic cell in the station can get the reactive power output of each unit through the global optimization. 


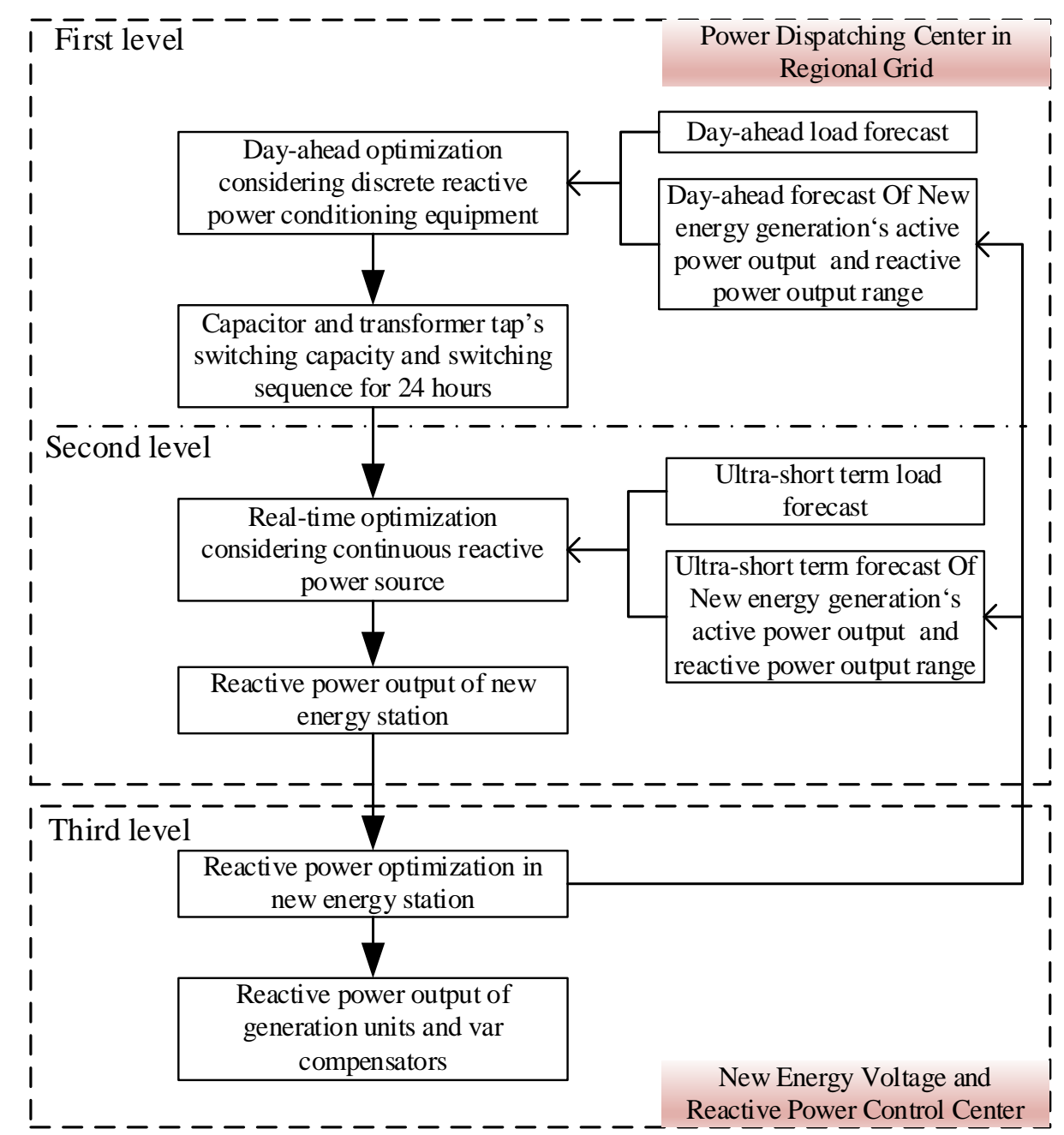

Fig.1. Three-level reactive power optimization model in regional grid

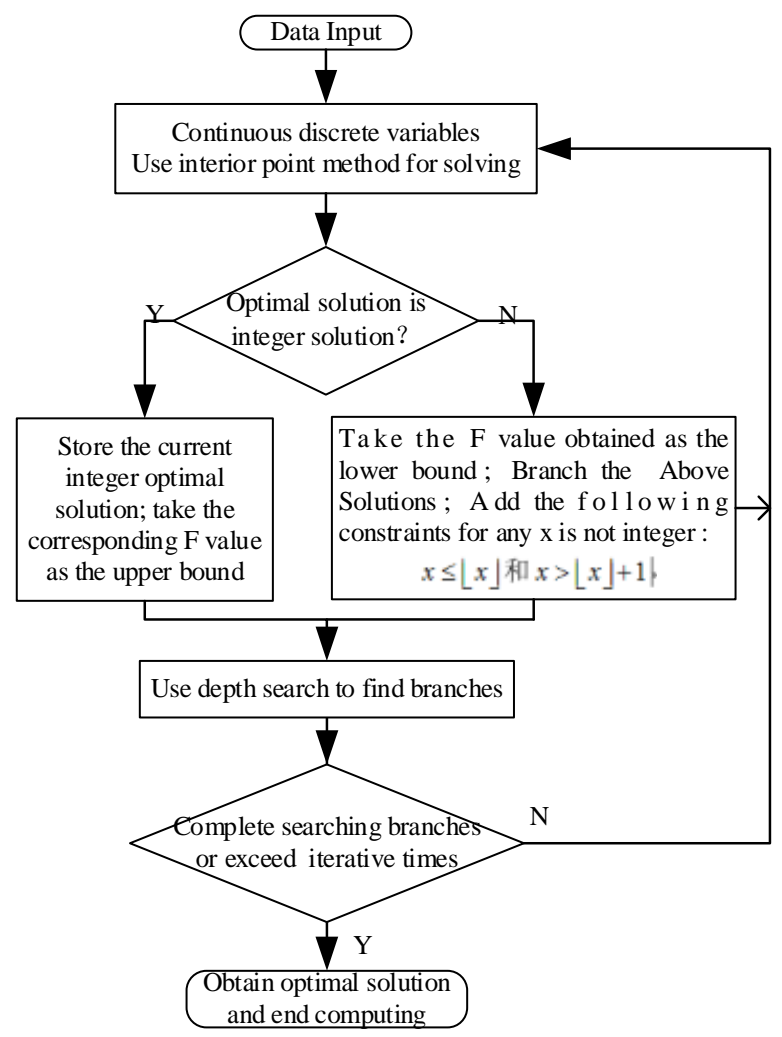

Fig.2 Optimization process of interior point method based on branch and bound method 


\section{Mathematical model of reactive power optimization}

\subsection{Object functions}

(1) Day-ahead reactive power optimization in grid

The day of the load is divided into 24 periods, and the total power loss in the next day is chosen as the objective function considering the economic operation of power grid:

$$
\min E=\sum_{t=0}^{23} P_{\text {loss }}=\sum_{t=0}^{23} \sum_{i=1}^{n} U_{i} \sum_{j=1}^{n} U_{j}\left(G_{i j} \cos \delta_{i j}+B_{i j} \sin \delta_{i j}\right)
$$

(2) Real-time reactive power optimization in grid

In order to ensure the voltage quality of the users, minimizing active power loss and voltage deviation are chosen as objective functions in the real-time optimization model:

$$
\min P_{\text {loss }}=\sum_{i=1}^{n} U_{i} \sum_{j=1}^{n} U_{j}\left(G_{i j} \cos \delta_{i j}+B_{i j} \sin \delta_{i j}\right)
$$

$$
\min \Delta U=\sum_{j=1}^{n}\left|U_{j}-U_{j}^{\text {ideal }}\right|
$$

(3) Reactive power optimization in new energy stations

In order to ensure the economic operation of new energy stations, the active power loss is chosen as objective function:

$$
\min f=P_{\text {ploss }}
$$

Where $\mathrm{E}$ is the regional grid's electric energy loss in the whole day; $P_{\text {loss }}$ is the active power loss in the regional grid; $\Delta U$ is the voltage deviation; $G_{i j}, B_{i j}$ and $\delta_{i j}$ are conductance, susceptance and phase angle difference between the $i$-th node and $j$-th node; $n$ is the total number of nodes; $U_{j}^{\text {ideal }}$ is the desired voltage of the $j$-th node; $P_{\text {ploss }}$ is the active power loss in the new energy station.

\subsection{Constraints}

1、 Normal constraints

Including power flow balance constraints, node voltage constraint and capacitor's switching capacity constraint.

2、Other constraints

The first-level and second-level optimizations belong to grid-level optimization which needs to consider examinational scope of power factor in the high voltage side of $220 \mathrm{kV}$ substations. Also the new energy station's reactive power output constraint should be considered:

$$
\cos \varphi_{\min } \leq \cos \varphi \leq \cos \varphi_{\max } \quad \text { (5) } \quad Q_{\mathrm{G} \min } \leq Q_{G} \leq Q_{\mathrm{G} \max }
$$

The limit of discrete equipment's action times within a day is considered in the grid's day-ahead reactive power optimization:

$$
\sum_{t=0}^{23}\left|Q_{t+1}-Q_{t}\right| \leq S_{Q} C_{Q}
$$

Where $C_{Q}$ is the capacitor banks' maximum allowed number of actions within a day; $S_{Q}$ is the adjustment step of capacitor banks' reactive power output.

In the third-level of new energy station's reactive power optimization, new energy stations' reactive power output should meet the requirements of grid scheduling:

$$
\left|Q_{\text {out }}-Q_{\text {ref }}\right| \leq \varepsilon
$$

where $Q_{\text {out }}$ is the reactive power in the new energy station exit; $Q_{\text {ref }}$ is the reactive power scheduling instruction from regional grid; $\varepsilon$ is assumed to be 0.0001 here.

Reactive power limit of wind turbines can be seen in reference [8] and reactive power limit of photovoltaic plants can be seen in reference [12].

\section{Coordinated optimization method based on interior point method}

Considering the practical engineering application, the next 24 hours is simplified as several decoupled optimization periods to process the limit of equipment action times, and the load of each cycle is the average load during this period. As the interior point method has a good convergence effect and fast calculation speed, and in order to deal with discrete variables, interior point method 
based on branch and bound method is adopted to solve the reactive power optimization problem of capacitors and new energy station's reactive power output. The detailed algorithm flowchart is shown in Fig.2.

\section{Case Study}

The case contains one $220 \mathrm{kV}$ substation and six $110 \mathrm{kV}$ substations; five capacitor banks are installed whose daily switching limit is five. The optimization is based on the active power dispatching results. Fig. 4 shows the daily predicted output curves of new energy stations and load.

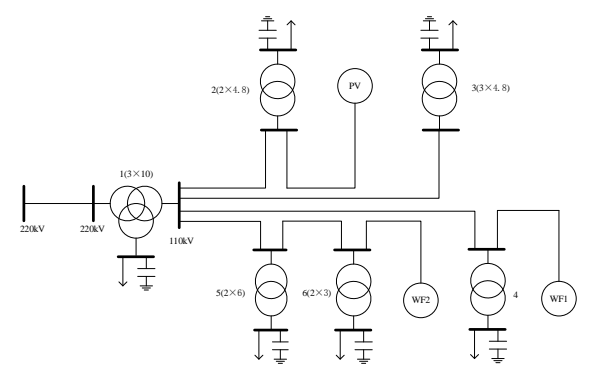

Fig.3 Electric diagram of district power network
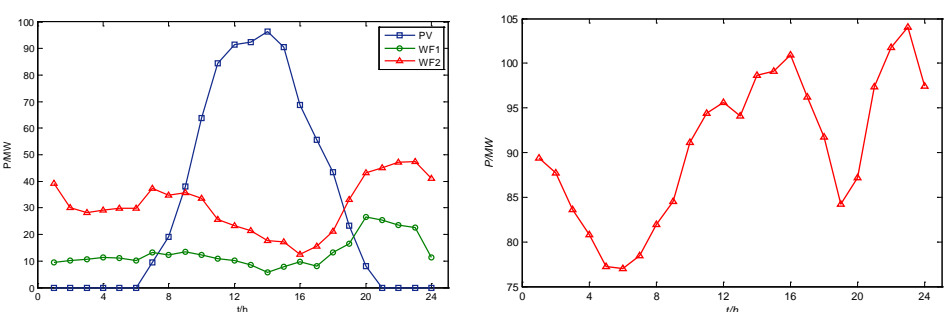

Fig.4 Daily predicted output curves of new energy stations and load

1、Day-ahead reactive power optimization results

Considering that the node voltage may approach the upper limit after power loss optimization, the upper limit in day-ahead reactive power dispatch is compressed to 1.055 from 1.07 in order to ensure sufficient voltage regulation margin in real-time scheduling.

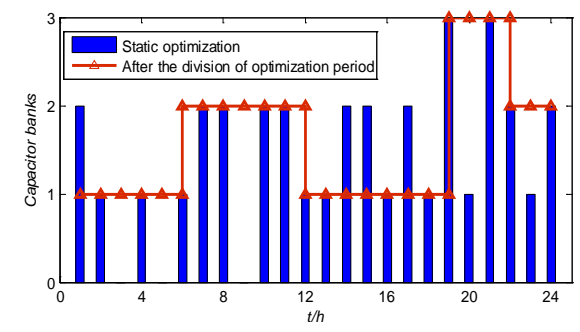

Fig.5 Capacitor switching results of substation 1

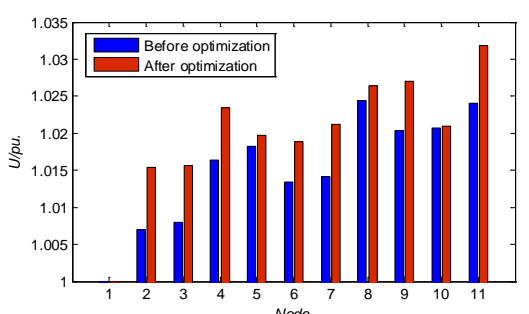

(a)

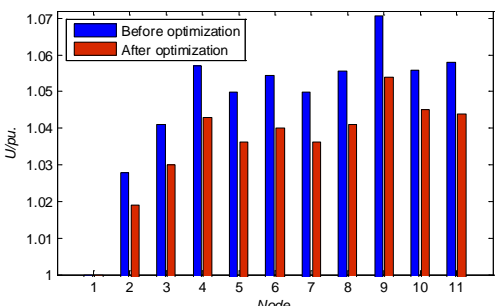

(b) station participating in real-time optimization

Fig. 5 shows the comparison of static optimization results and optimization results after the division of optimization period. As the result shows, the switching frequency is significantly reduced and within the range. In addition, the amount of computations is much smaller.

2、Real-time reactive power optimization results

Two cases are analyzed according to the day-ahead results:

a. Normal case: Suppose little changes in load and output than predicted.

b. The worst case: Suppose large changes in load and output, and the voltage will exceed the upper limit according to day-ahead results.

The active loss and voltage deviation results before and after optimization are shown in Table 1.

Table 1 Results before and after new energy station participating in real-time optimization

\begin{tabular}{ccccc}
\hline \multirow{2}{*}{ Objective } & \multicolumn{2}{c}{ Case a } & \multicolumn{2}{c}{ Case b } \\
\cline { 2 - 5 } & Before & After & Before & After \\
\hline Ploss/MW & 1.237 & 1.132 & 5.868 & 5.927 \\
$\Delta \mathrm{U} / \mathrm{kV}$ & 0.1305 & 0.2263 & 0.2836 & 0.1653 \\
\hline
\end{tabular}

Table 2 Output of new energy stations

\begin{tabular}{cccc}
\multicolumn{5}{c}{ Table 2 Output of new energy stations } \\
\hline Stations & PV & WF1 & WF2 \\
\hline Q/MVar & -2.07 & -0.52 & 2.26
\end{tabular}

From the results in Table 1, the active power loss after optimization in case(a) drops $8.49 \%$; the voltage is increased as can be seen in fig.6(a). In both cases, the voltage can be guaranteed within 
the operating range. Though the power loss in case(b) is increased, it's increased by only $0.49 \%$, indicating that safety and economical efficiency are both maintained. The results show that taking renewable power plants as continuous reactive power adjustment means can effectively improve the economy of the grid and it is necessary and valid to take the active loss and voltage deviation minimization into account in real-time optimization model.

3、 Reactive power optimization results of new energy station

Based on the result in the scene of 2.a, the reactive power output of each station is shown in Table 2. Take wind farm 1 as an example to verify the optimization model inside the station. If use the traditional proportional allocation method, the power loss of the station is $2.258 \mathrm{MW}$, but it can't guarantee the reactive power at the exit essentially matching the dispatch instruction. However, the model proposed in this paper can achieve the dispatch instruction and reduce the power loss to 2.137MW. Thus the rationality and effectiveness of the proposed optimization model is proved.

\section{Conclusion}

On the basis of taking new energy station as continuous reactive power adjustment means, a three-level dynamic reactive power optimization model for district power network with large scale new energy is built from different time scales. Different objective functions are constructed and different decision variables with different output characteristics are processed in each level according to different needs. The proposed three-level coordinated model is implemented in a real district power network and the results show that it can give full play to the own characteristics of capacitors and new energy generators, achieve their coordinated optimal voltage control under different circumstances, and effectively reduce the active power loss of the district power grid and new energy stations thus improving the economy and security of the grid.

\section{References}

[1]. Zhao Jingjing, Fu Yang, Li Dongdong. Reactive Power Optimization in Distribution Network Considering Reactive Power Regulation Capability of DFIG Wind Farm.[J].Automation of Electric Power System, 2011, 35(11): 33-38.

[2]. He Yuqing, Peng Jianchun, Mao Lilin, Cao Lihua. Reactive Power Optimization in Distribution System with Multiple Wind Power Generators.[J].Automation of Electric Power System, 2010, 34(19): 37-41.

[3]. Cai Changchun, Ding Xiaoqun, Wang Kuan, Shen Maoya, Wu Zhen. Simplified Method of Dynamic Reactive Power Optimization and Its Implementation [J]. Automation of Electric Power System, 2008, 32(5): 43-46.

[4]. Guo kang. Research on Reactive Power Optimization of Distribution Network with PV Power Station [D].North China Electric Power University,2012.

[5]. Zhao Ligang, Fang Dazhong, Kong Xiangyu, Hou Youhua. A strategy of reactive power control for wind farm operation using SVC and DFIG [J]. Power System Protection and Control, 2012, 40(2). 